\title{
MATERIAL DIDACTICO PARA LA LECTOESCRITURA EN ESTUDIANTES CON DISCAPACIDAD AUDITIVA
}

\section{TEACHING MATERIALS FOR LITERACY FOR STUDENTS WITH HEARING IMPAIRMENT}

\section{MATERIAL DIDÁTICO PARA A LECTOESCRITURA EM ESTUDANTES COM DEFICIÊNCIA AUDITIVE}

Jaime Franco BracamonteUniversidad del Atlántico jrafaelfranco@est.uniatlantico.edu.co

Barranquilla-Colombia

Shirley Barreiro Novoa

Universidad del Atlántico

shirlybarreiro@mail.uniatlantico.edu.co

Barranquilla-Colombia

Diagramación

Sindy Catherine Charcas Ibarra

Fotografia portada

Santiago Ardila Acero

Encuentre este artículo en:

http://revistas.uniminuto.edu/index.php/IYD

Para citar este artículo / To cite this article

Franco, J. \& Barreiro, S. (2021). Material didáctico para la lectoescritura en

estudiantes con discapacidad auditiva. Inclusión \& Desarrollo, 8(1), pp 6-27
Fecha de recepción: 1 de abril de 2020

Fecha de aceptación: 24 de octubre de 2020

Fecha de publicación: 1 de enero de 2021

\section{RESUMEN}

En el presente articulo producto de una investigación donde se expone el nivel de las habilidades de lectoescritura que tienen jóvenes con discapacidad auditiva, argumentado bajo fundamentos teóricos y comprobados en el resultado de un análisis de observación en una Muestra de estudiantes con esta discapacidad, que tuvo como resultado la creación de una propuesta educativa inclusiva, la cual es el Kamishibai como estrategia didáctica multimedia para fortalecer la lectoescritura en esta población. Objetivo: Fortalecer la lectoescritura mediante el Kamishibai como material didáctico multimedia que fortalezca el proceso de lectoescritura en estudiantes con discapacidad auditiva. Metodología: esta investigación, se enmarca en el paradigma interpretativo contextualizado bajo la investigación cualitativa, haciendo alusión a caracteres, atributos no cuantificables que pueden describir la situación, para así comprender o explicar el fenómeno observado en los estudiantes con discapacidad auditiva en contextos sociales y académicos. Resultados: En esta investigación se ha observado que existe de forma general una problemática desde el que hacer pedagógico en la estimulación y enseñanza de la lengua escrita en estudiantes sordos. Es así como el Kamishibai adaptado a la población con discapacidad auditiva, 
pretende fortalecer el proceso de lectoescritura en los estudiantes sordos, proporcionando esta herramienta didáctica a los docentes para ejecutarlo de manera creativa a los jóvenes, favoreciendo la interacción de estos con el entorno. Conclusiones: Se evidenciada la debilidad que existe en el sistema educativo colombiano en cuanto a estrategias o metodologías para la enseñanza de la lengua escrita en estudiantes sordos. Desde el rol de educador especial estimular competencias básicas para la persona sorda y así brindarles una mejor oportunidad de comunicación al interactuar fácilmente a través de la lectura y escritura con su entorno.

Palabras claves: persona sorda, discapacidad auditiva, Sódori, Kamishibai, lectoescritura, didácticas, lengua de señas.

\section{ABSTRACT}

In this article, the product of an investigation that exposes the level of literacy skills that young people with hearing disabilities have, argued on theoretical grounds and verified in the result of an observation analysis in a sample of students with this disability, which resulted in the creation of an inclusive educational proposal, which is the Kamishibai as a multimedia didactic strategy to strengthen literacy in this population. Objetive. Strengthen literacy through Kamishibai as a multimedia educational material to strengthen the literacy process in students with hearing disabilities. Methodology. This research, is framed in the interpretive paradigm contextualized under qualitative research, alluding to characters, non-quantifiable attributes that can describe the situation, to understand or explain the phenomenon observed in students with hearing impairment in social and academic contexts. Results. In this research it has been observed that there is a general problem from which to make pedagogical in the stimulation and teaching of the written language in deaf students. This is how the Kamishibai adapted to the hearing-impaired population, aims to strengthen the literacy process in deaf students, providing this didactic tool to teachers to creatively execute it to young people, encouraging the interaction of these with the environment. Conclusions. The weakness that exists in the Colombian education system in terms of strategies or methodologies for the teaching of the written language in deaf students is evident. From the role of special educator stimulate basic competencies for the deaf person and thus provide a better opportunity for communication by interacting easily through reading and writing with their environment.

Key Words: deaf person, hearing impairment, Sódori, Kamishibai, literacy, didactics, sign language.

\section{SUMÀRIO}

No presente artigo produto de uma pesquisa onde se expõe o nível das habilidades de lectoescritura que têm jovens com deficiência auditiva, argumentado sob fundamentos teóricos e comprovados no resultado de uma análise de observação numa Amostra de estudantes com esta deficiência, que teve como resultado a criação de uma proposta educativa inclusiva, que é o Kamishibai como estratégia didática multimídia para fortalecer a lectoescritura nesta população. Alvo. Fortalecer a lectoescritura mediante o Kamishibai como material didático multimídia que fortaleça o processo de lectoescritura em estudantes com deficiência auditiva. Metodologia. Esta pesquisa, enquadra-se no paradigma interpretativo contextualizado sob a investigação qualitativa, fazendo alusão a caracteres, atributos não quantificáveis que podem descrever a situação, para assim compreender ou explicar o fenômeno observado nos estudantes com deficiência auditiva em contextos sociais e acadêmicos. Resultados. Nesta pesquisa tem-se observado que existe de forma geral uma problemática desde o que fazer pedagógico na estimulação e ensino da língua escrita em estudantes surdos. É assim como o Kamishibai adaptado à população com deficiência auditiva, pretende fortalecer o processo de lectoescritura nos estudantes surdos, proporcionando esta ferramenta didática aos docentes para executá-lo de maneira criativa aos jovens, favorecendo a interação destes com o ambiente. Conclusões. É evidenciada a debilidade que existe no sistema educativo colombiano quanto a estratégias ou metodologias para o ensino da língua escrita em estudantes surdos. Desde o papel de educador especial estimular competências básicas para a pessoa surda e assim proporcionarIhes uma melhor oportunidade de comunicação ao interagir facilmente através da leitura e escrita com seu entorno.

Palavras chave: pessoa surda, deficiência auditiva, Sódori, Kamishibai, lectoescritura, didácticas, língua gestual. 


\section{Introducción}

En la actualidad existen tendencias educativas de orden mundial, las cuales suponen desafíos para el sistema educativo, aún más, cuando se reconoce que al hablar de educación es necesario abordar el tema de diversidad, es por esto que la UNESCO (2008) define la educación inclusiva como "El derecho de todos los alumnos a recibir una educación de calidad que satisfaga sus necesidades básicas de aprendizaje y enriquezca sus vidas".

En cuanto a este concepto, el Ministerio de Educación Nacional (2008) citado por Lugo en 2019, plantea que es una apuesta por "atender con calidad y equidad las necesidades comunes y específicas que presentan los estudiantes" (p.8).

Para la UNESCO (2008), citado por Rodríguez (2020), la educación inclusiva debe caracterizarse por prestar especial atención a los grupos marginales y vulnerables dentro de estos grupos se encuentran los alumnos con discapacidad a los cuales se les debe ofrecer el apoyo y refuerzo necesario para que se integren lo mejor posible.

Esta investigación se enfoca en estudiantes con discapacidad auditiva los cuales se caracterizan por pertenecer a una comunidad minoritaria lingüística con capacidades diferentes usuarios lengua de señas, por tratarse de una lengua natural surgida en el seno de la comunidad sorda, cuyas características visogestuales facilitan una rápida adquisición, la comunicación fluida entre sus hablantes $y$ el desarrollo de actividades cognitivas. En relación con la segunda lengua (español), es relevante hacer una distinción entre la oralidad y la escritura. Para las personas sordas, la posibilidad de comunicarse por la vía auditivaoral suele presentar grandes dificultades. En cambio, la lectura y la escritura -por ser ambas de naturaleza visual- podrían alcanzar índices similares de apropiación en oyentes y en sordos.

Cobra mayor importancia si el aprendizaje de la lectoescritura, se convierte en fuente de conocimientos y en herramienta de comunicación.

La investigación que se presenta a continuación pretende contribuir a la atención de uno de los problemas que más aquejan a las instituciones que tienen incluido a estudiantes sordos. Una problemática que se hace latente durante toda la estancia escolar de cada niño o joven sordo, haciendo menos interesante el proceso formativo y aumentando aún más la barrera de comunicación con sus pares sin discapacidad y también con todo su entorno, sea familiar o social. La carencia de habilidades para leer y escribir que presentan la mayoría de personas sordas hoy día, se debe a la poca atención que se le brinda en la escuela a este tema. Es precisamente el abordaje que hace esta investigación, identificar realmente el problema, fundamentándose en teóricos y teniendo en cuenta otras experiencias académicas de la enseñanza de la lectura y escritura en otros países y seleccionando una serie de actividades que se desarrollaran mediante una Herramienta virtual interactiva para así fortalecer este proceso lectoescritor.

Desde la escuela se debe optar por modelos pedagógicos que seadaptenalas características del alumnado y, por tanto, desplegar todas las ayudas que favorezcan el aprendizaje de la lectoescritura en los niños y niñas sordos.

En Colombia existe un gran número de personas sordas analfabetas de 15 años en adelante, según un estudio realizado por el DANE junto al MEN, se encontró que en Colombia hay 504.083 personas sordas, de las cuales 174.919 no saben leer ni escribir. (El Observatorio Social - Población Sorda 
Colombiana del INSOR, 2017).

Frente a este panorama, estudios muestran que los docentes carecen de herramientas y conocimientos idóneos para atender a estos estudiantes; además que las diferencias lingüísticas, contextuales, sociales, no favorece. $Y$ los docentes carecen de un perfil bilingüe para estar al frente de esta población.

Estos estudiantes sordos al ver las carencias que hay, no demuestran interés a la lengua escrita, tienen bajo rendimiento académico, no saben leer ni escribir o hasta deciden por no ir más al colegio. Por lo cual surge una pregunta problema:

¿Cómo fortalecer la lectoescritura a estudiantes con discapacidad auditiva mediante un material didáctico multimedia?

En la etapa escolar es tan importante el aprendizaje de nociones matemáticas, la adquisición de conocimientos en historia, en geografía, entre otras disciplinas, de esta manera se garantiza el desarrollo intelectual potencial de los estudiantes.

\section{Estado del Arte}

Las investigaciones que giran alrededor de este tema, pueden dejar muy claro que se han hecho intentos por mejorar la calidad educativa en cuanto a lectura y escritura de estudiantes sordo.

Inicialmente, Tovar (2000), Citado por Tobar, L. (2017), menciona que "para el sordo, la lengua escrita debe ser vista como una segunda lengua necesaria para su supervivencia en una sociedad compleja en la cual la lengua escrita juega un papel clave". Además, como una oportunidad de crecimiento personal fundamental para su éxito académico, es en sí, una herramienta para que se realice como ser humano en iguales condiciones a la de los oyentes.

Por otra parte, Liaño (2018), expresa que "La lectoescritura es un proceso complejo que se lleva a cabo en el ámbito educativo y que precisa de diversas habilidades para poder ponerla en práctica"

Afirmaciones que nos invitan a reflexionar en la problemática de este trabajo de investigación y la importancia que tiene la lectoescritura para el desarrollo integral de cualquier individuo, es así como se puede concordar con las distintas afirmaciones sobre el bajo nivel de dominio de la lengua escrita que posee la mayoría de personas sordas en el mundo y está respaldada por investigaciones realizadas tanto en los países desarrollados como en los subdesarrollados.

No se trata "de que se les esté imponiendo a los sordos una obligación académica sólo por asimilarlos a la sociedad dominante de oyentes, sino que se ve la urgencia de proveerlos de una herramienta que les permita un desarrollo humano en igualdad de 5 condiciones que sus compatriotas oyentes, tal como lo ordena la Constitución Nacional" (Tovar, 2000: 74) Tanto la adquisición del lenguaje como de la lengua en el ser humano es tan vital para convivir en sociedad, para la comunicación; en el caso de estudiantes con discapacidad auditiva, no solo basta con saber Lengua de Señas, debido a que se habla hoy día de una educación bilingüe, bicultural.

De acuerdo a lo anterior, Morales (2008) citado por Morales G (2015), indica "que la lengua oral y la de señas no constituyen una oposición, sino dos canales diferentes e igualmente eficientes para la transmisión y recepción del lenguaje. Conjeturas que soportan la ventaja de la persona sorda en el aprendizaje de la segunda lengua. 
En este orden de ideas, el uso de estas herramientas permite una apertura al mundo y mayores posibilidades de integración para las personas con discapacidad auditiva. (Koon, R. y de la Vega, M.E., 2000 citado por Silva Lascano, C. A. (2017).

Por tanto, se hace necesario adquirir y fortalecer habilidades en la lectoescritura y así vivir una verdadera inclusión educativa.

Fortalecer la seguridad al comunicarse y enfrentar a un grupo. De un recurso pedagógico y didáctico, da oportunidades para ampliar la mirada multicultural, despertar el deseo de crear relatos, disfrutarlos en grupo, abordar diversidad de obras, hacer integración curricular entre distintos saberes propios de subsectores como la educación artística, la música, la educación tecnológica, etc. Además, otorga protagonismo tanto al profesor como al alumno, quien puede ser espectador del relato $o$ activo narrador.

Los nuevos ámbitos educativos que exigen las sociedades de la información y el conocimiento, destacan la importancia de repensar los elementos básicos de los procesos de enseñanza y aprendizaje, de ahí la necesidad de transformar estos en un marco de acción donde el educando, aprenda a aprender, a hacer, a ser y a convivir, exigiéndose el acompañamiento responsable de procesos de aprendizaje, el desarrollo permanente de habilidades cognitivas y la implementación de nuevas tecnologías como agentes externos de alta importancia educativa y garantes de un enfoque equitativo, pertinente e inclusivo.

Según el portal, Eduteka (2007), citado por Valdez, M. D. R. G. (2020), muestra otra nueva razón que evidencia la necesidad de incorporar las TIC en los procesos educativos, tiene que ver con el hecho de que la gran mayoría de escuelas y colegios de Latinoamérica, son carentes de recursos educativos, lo cual se traduce en la escasez de materiales en sus bibliotecas, siendo esta falencia a su vez, una de las mayores limitaciones para la formación inclusiva de niños y jóvenes de los sectores menos favorecidos económicamente.

Por su parte, Henao Ramírez y Medina (2001), citado por Agudelo (2012), reporta una investigación realizada en Medellín con treinta estudiantes sordos entre 13 y 18 años de edad, el propósito principal fue contribuir a cualificar la educación ofrecida a la población sorda para el desarrollo de habilidades de lectura funcional, apoyada en un entorno multimedial, materiales y experiencias comunicativas estimulantes en lengua de señas colombianas y en lengua escrita.

El grupo experimental utilizó en su instrucción un entorno multimedial llamado Lectura funcional para sordos, con su respectivo módulo de actividades complementarias, mientras que el otro grupo (control), no usó este tipo de herramientas TIC. Se aplicaron dos pruebas, una de competencia en lengua de señas colombiana y otra en competencia lectora durante dos momentos diferentes, como pre test donde no se encontraron diferencias significativas en los desempeños y como posttest.

Durante cuatro meses los alumnos sordos del grupo experimental participaron en la propuesta didáctica realizada en un laboratorio de informática de la Universidad de Antioquia, la cual tuvo una intensidad de dieciséis horas semanales. En el análisis realizado al desempeño de este grupo durante la participación en el programa multimedial y en el post-test se encontraron algunos hallazgos:

Se evidenció mayor habilidad para buscar información en diccionarios, libros de poemas y directorios telefónicos, en los alumnos del 
grupo experimental frente a sus compañeros del grupo control, la eficacia para responder acertadamente ítems que indagan por fecha, nombre y apellidos, ciudad, descendencia, etc., fue significativa para el grupo experimental, la habilidad para reconocer palabras y organizarlas en un conjunto de frases con sentido coherente, fue mayor para el grupo experimental, la capacidad para seguir instrucciones escritas, así como para asociar símbolos de uso cotidiano con sus respectivas palabras escritas, es mayor en los estudiantes del grupo que usó el entorno multimedial frente a sus compañeros del grupo control, la capacidad para responder correctamente preguntas de comprensión lectora así como para seleccionar las palabras que completan adecuadamente un conjunto de frases es significativamente mayor para el grupo experimental, el desempeño logrado al explicar en lengua de señas un conjunto de frases es significativamente mejor para los estudiantes del grupo experimental, de manera general, los resultados del post test, arrojaron un desarrollo de habilidades comunicativas más eficaz en lengua de señas y comprensión lectora para los estudiantes del grupo experimental., quienes reconocieron estimulación del entorno multimedial.

Las instituciones escolares tienen como objetivo principal iniciar el proceso de enseñanza de la lectura y escritura de los niños en los primeros años de vida, de esta manera es que la adquisición y desarrollo del lenguaje ayuda a desarrollar las competencias básicas de comunicación en los estudiantes.

De acuerdo a estudios anglosajones, una investigación hecha en la Pontificia Universidad Católica de Chile, señalan que la habilidad lectora general en alumnos con déficit auditivos permanece muy por debajo de la de los estudiantes oyentes (Allinder \& Eccarius 1999; Schimmel, Edwards \& Prickett 1999; Musselman 2000). Schimmel, Edwards y Prickett (1999), citado por Solano, C. I. H., \& Barraza, J. A. V. (2018), reportan que estudiantes de 18 años tienen un desempeño lector promedio equivalente al observado en el cuarto grado en la escuela norteamericana.

En los países de habla española la situación es similar, lo cual se ve confirmado por un estudio realizado en Chile, en el que se reporta que los niños sordos chilenos se encuentran por debajo de lo esperado para su edad en tareas de lectura y escritura, presentando dificultades en la adquisición de habilidades de lectura funcional (Lissi et al. 2003 citado por Sosa,2018).

La mayoría de los autores concuerda en señalar que la pobreza de vocabulario en los sordos se relaciona con el grado de pérdida auditiva y se correlaciona con las habilidades de lectura. Los investigadores explican que los problemas de lectura de los sordos pueden ser producto del desconocimiento del significado de las palabras que aparecen en el texto escrito, que estos lectores se fijan más en las palabras familiares y usan más sustantivos concretos y verbos familiares que palabras abstractas.

Asimismo, ya que su vocabulario es limitado presentan pobres expresiones escritas. Los autores concluyen que el conocimiento de vocabulario es un indicador de las habilidades en lectura, que la falta de vocabulario no puede ser compensada ni sintáctica ni semánticamente, que el desempeño de los sordos es mejor con palabras con un solo significado y, finalmente, que los sordos que poseen un lenguaje fluido y natural desde el nacimiento obtienen mejores resultados en las pruebas de conocimiento de vocabulario que los sordos que no tienen un lenguaje consolidado.

De esta manera esta investigación tiene como propósito; Fortalecer el proceso de lectoescritura en estudiantes con discapacidad 
auditiva mediante el Kamishibai como material didáctico multimedia. Para alcanzar este meta se trazó una ruta en la cual se tuvo en cuenta inicialmente: Determinar los fundamentos teóricos de herramientas virtuales y el proceso lectoescritor en estudiantes con discapacidad auditiva. Seguidamente Identificar las dificultades del proceso lectoescritor de los estudiantes con discapacidad auditiva de media secundaria en una escuela de la ciudad de Barranquilla - Colombia llamada "Salvador Suárez Suárez". Sucesivamente se adaptó el Kamishibai como herramienta para fortalecer el proceso lectoescritor de los estudiantes con discapacidad auditiva y por último se aplicó el Kamishibai.

\section{Marco teórico y referentes conceptuales Discapacidad auditiva}

Se define desde la perspectiva socio antropológica que menciona el MEN mediante el Grupo de Investigación y Desarrollo Pedagógico INSOR (2006), citado por Bernal Velázquez. A.L. (2018), desde la que los sordos son considerados como personas con plenas capacidades para desarrollarse integralmente; se reconoce y respeta que adquieren y usan la lengua de señas como primera lengua; conforman una comunidad lingüística minoritaria y dicha comunidad se denomina comunidad sorda. Por otra parte, la sordera es concebida como una experiencia visual antes que como una deficiencia auditiva.

La experiencia visual hace referencia a las formas particulares de procesamiento de la información que tienen los sordos al percibir el mundo prioritariamente desde el canal visual, lo que incluye todo tipo de significaciones, representaciones y/o producciones en el campo intelectual, lingüístico, ético, estético, artístico, cognoscitivo, etc. Ser sordo, nacer sordo ubica a un individuo en una situación extraordinaria con una gama de posibilidades lingüísticas y en consecuencia intelectuales y culturales para la expresión y comprensión del mundo.

\section{Kamishibai}

Teatro de papel, conjunto de láminas con dibujo llamativos, sin texto, y que en el reverso traen escrito el relato ilustrado en las imágenes. Predominan los textos narrativos, como fábulas, cuentos, leyendas, pero también es posible abordar contenidos de aprendizaje en un texto de carácter informativo.

Imagen 1: Kamishibai adaptado

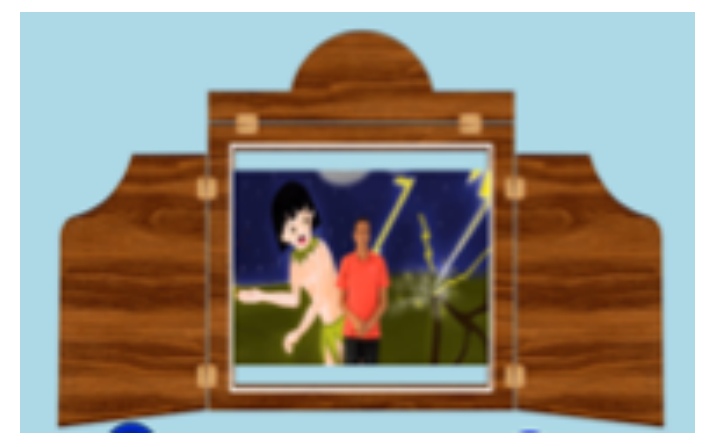

Fuente: elaboración propia (Imagen 1).

Incluye láminas con dibujos grandes diseñados con delineaciones simples y atractivas, esto junto al cuento que se va leyendo, hace que con el Kamishibai se origine un ambiente mágico y de concentración en torno a la historia que se está contando, pero además permite que niños y niñas lean tras el teatrillo en voz alta adquiriendo confianza, de esta manera la técnica se transforma en un andamiaje para fortalecer la seguridad al comunicarse y enfrentar a un grupo. Como recurso pedagógico y didáctico, da oportunidades para ampliar la mirada multicultural, despertar el deseo de crear relatos, disfrutarlos en grupo, abordar diversidad de obras, hacer integración curricular entre distintos saberes propios de subsectores como la educación artística, la música, la educación tecnológica, etc. Además, otorga protagonismo tanto al profesor como al 
alumno, quien puede ser espectador del relato $o$ activo narrador.

Es sabido que los niños y niñas que escuchen relatos, cuentos, poesías desde edades tempranas, ampliarán su vocabulario, sofisticarán el manejo de la sintaxis oral y mejorarán su predisposición a la producción escrita. El principio es muy simple y claro, los niños y niñas auditores, narradores o lectores de narraciones se enfrentan a palabras conocidas en nuevos contextos, y además multiplican su léxico con los nuevos conceptos que emergen de los relatos.

Al respecto, Lilia Concha Carreño de la Universidad Alberto Hurtado en Chile, menciona después de intervenir en un grupo de estudiantes, que el Kamishibai ofrece la oportunidad de abrir diálogo y conversación posterior a la narración, propicia las relaciones sociales, favorece la comunicación oral, ayuda a crear un ambiente de confianza, acogedor y lúdico que motiva la expresión personal. "De los diversos instrumentos del hombre, el más asombroso es sin duda, el libro. Los demás son extensiones del cuerpo. El microscopio, el telescopio, son extensiones de su vista; el teléfono es una extensión del oído y la voz; el arado y la espada, extensiones de su brazo. Pero el libro es otra cosa: el libro es una extensión de la memoria y la imaginación" (Jorge Luis Borges, 1980. Citado por Gómez, H. M. R. (2017). El Kamishibai al igual que el libro, es un objeto cultural que ayuda a prolongar el pensamiento, la imaginación, la memoria, el relato, la reflexión, el espíritu de las vidas que alimentan las narraciones, las historias que transitan y habitan en el teatro de papel.

\section{Material multimedia}

Siendo esto muy valioso por el gran impacto que producen tales como las imágenes, la interactividad, la posibilidad de desarrollar actividades a medida de las necesidades, los diferentes modos de comunicación (mediata o inmediata) y el particular atractivo que todos estos elementos representan para los niños y jóvenes, convierte a las TIC en instrumentos complementarios importantísimos, que pueden enriquecer y acelerar los procesos de apropiación; según Pérez Marqués (1999), citado por Yovera Sandoval, E. C. (2018), "Los materiales didácticos informáticos constituyen un recurso formativo complementario que debe utilizarse de la manera adecuada y en los momentos oportunos".

Cabero (2000), conceptúa que las Tecnologías de la Información y la Comunicación (TIC), son utilizadas socialmente para referirse a una serie de nuevos medios como los hipertextos, los multimedios, Internet, la realidad virtual o la televisión por satélite. El mismo Cabero, señala que dichas tecnologías tienen un carácter de interactividad en torno a las telecomunicaciones, la informática educativa, los audiovisuales, y gran cantidad de recursos multimedia. Cuando se articula este tipo de tecnologías al contexto educativo, se habla de las TIC en educación, se destaca como un importante valor, que tales tecnologías permiten el acceso, desarrollo, tratamiento y procesamiento de la información, sin el límite condicionante que imponen las barreras espacio temporales y con el beneficio adicional de potenciar en buena medida, la interactividad y el asincronismo entre los actores educativos de cada plantel formativo. Esa característica de asincronismo y eliminación de barreras espacio temporales que impregnan las TIC en educación, son muestra fehaciente de la manera como William de Jesús Mesa Agudelo 63 No. 7 / diciembre / 2012 ellas, pueden contribuir a disminuir, además de la enorme brecha digital entre estudiantes de distintos pueblos, la inequidad y la exclusión educativa a que están sometidos gran cantidad de estudiantes, que por su condición física o por su diversidad cultural y social, no pueden 
gozar en igualdad de condiciones de algunos de sus derechos como el acceso a las fuentes de conocimiento, al aprendizaje y a la educación.

En la investigación hecha por Patricia M. Folco (2010), citado por Choez y Arreaga (2019), señala que el ámbito escolar debe propulsar la utilización de los recursos de información y comunicación para la construcción de significado. Esto incluye además de los juegos, otras herramientas tales como: procesadores de textos, programas de presentaciones, editores de imagen, planillas de cálculo, software educativo con fines específicos (Cataldi, Z.; Lage, F.; Pessacq, R. y García Martínez, R., 1999 citado por Zúñiga Palma, M. J. (2018), y herramientas de Internet tales como navegadores, buscadores, programas de mensajería, entre otros.

\section{Metodología}

La investigación se realizó en una de las 10 instituciones seleccionadas por el INSOR a nivel Nacional, para ser piloto del proyecto que lidera esta entidad con el apoyo de MEN, "Colombia Primera en Educación Para Sordos", se trata de la Institución Educativa Distrital Salvador Suárez Suárez, ubicada en la Calle 52 No. 2075 Barranquilla-Atlántico de Colombia.

Se contó con una población de 20 estudiantes sordos, ubicados en los grados 10 y 11 de educación Básica secundaria .

Desde el punto de vista metodológico, se apoyó en el principio de que cualquier intento por definir una política educativa para sordos debe ajustarse a la visión, necesidades e intereses de la población a quien va dirigida y a la realidad que imponen todos los actores legítimamente involucrados en el problema. Como consecuencia de lo anterior, aparece la urgencia de un análisis de los procesos que intervienen en el proceso lecto-escritor entre las partes, la participación y la implementación de nuevas herramientas.

Siendo así, que el presente estudio de investigación, según lo que dice Heidegger, mencionado por Daniel Innerarity (2006) Se refiere a la interpretación de la Interacción Social, en la que propone que se deben estudiar las interpretaciones y significados que las personas le dan cuando interactúan, en distintas situaciones y la realidad social en la cual viven, esto es constante y los significados que se le otorgan pueden modificarse por otros, estos cambios son importantes para el interaccionismo simbólico, por tanto se relaciona con el paradigma interpretativo contextualizado bajo la investigación cualitativa, Cerda (2011), quien refiere que la investigación cualitativa hace alusión a caracteres, atributos o facultades no cuantificables que pueden describir, comprender o explicar los fenómenos sociales o acciones de un grupo o del ser humano.

Siendo así, que el presente estudio de investigación, según la teoría de Heidegger, "Se refiere a la interpretación de la Interacción Social" en la que propone que se deben estudiar las interpretaciones y significados que las personas le dan cuando interactúan, en distintas situaciones y la realidad social en la cual viven, esto es constante y los significados que se le otorgan pueden modificarse por otros, estos cambios son importantes para el interaccionismo simbólico, por tanto se relaciona con el paradigma interpretativo contextualizado bajo la investigación cualitativa, Cerda (2011), quien refiere que la investigación cualitativa hace alusión a caracteres, atributos o facultades no cuantificables que pueden describir, comprender o explicar los fenómenos sociales o acciones de un grupo o del ser humano. 
En cuanto al paradigma, es en donde la tarea del investigador, se traslada desde el análisis de la observación en un contexto bajo ciertos criterios, hasta llegar a una interpretación de la situación objeto de investigación. Es por ello, que el docente en formación se encamina con el trabajo investigativo hacia la solución del problema latente en los estudiantes sordos, a partir de la auto-reflexión para converger con este paradigma en el análisis de los sucesos en el aula de clase, otorgándole a la investigación, un carácter igualmente auto reflexivo, emancipatorio y transformador.

A su vez, se toma la investigación descriptiva, teniendo en cuenta en este caso y lo que menciona Tamayo, M. (2004). El proceso de la investigación científica. Editorial Limusa, el docente trabaja sobre realidades de hecho, presentes durante el paso de la vida académica de los estudiantes, en relación a la investigación en curso, aplica al tener en cuenta lo que presenta el grupo de colegiales con discapacidad auditiva en los grados de básica media; y su característica fundamental es la de presentar una interpretación correcta de los hechos dentro del salón de clases, evidenciados durante el proceso de cada fase de la investigación.

Lo anterior expuesto, responde al goce de libertad frente al diseño metodológico, que en el desarrollo de la investigación se construye mediante la Acción-Participación, que según Antonio Latorre (2007, p. 28) se destaca de otros métodos en la investigación cualitativa, la manera como se aborda el objeto de estudio, las intencionalidades o propósitos, el accionar de los actores sociales involucrados en la investigación, los diversos procedimientos que se desarrollan y los logros que se alcanzan.

Y además Latorre menciona, que las metas de la investigación-acción son: mejorar y/o transformar la práctica social y/o educativa, a la vez que procurar una mejor comprensión de dicha práctica, articular de manera permanente la investigación, la acción y la formación; acercarse a la realidad vinculando el cambio y el conocimiento.

Entre los instrumentos utilizados fueron: Observación directa, Entrevista Semiestructurada a los sujetos participantes (estudiantes sordos y docentes) y una prueba diagnóstica a los estudiantes implicados

Observación. Percepción + interpretación. Este es un proceso deliberado y sistemático que debe estar orientado por una pregunta o propósito, es necesario conocer el qué, el quién, el cómo y el cuándo se observa. (Cuadernos de Investigación, Metodología de la Investigación Instrumentos de recolección de información en investigación cualitativa, msc. Bessy Dolores Hernández, mayo 2009)

Este instrumento se pretende usar en las clases que reciben los estudiantes con discapacidad auditiva de Lenguaje.

1. Seguimiento permanente del desarrollo de los estudiantes en clase de lectura

2. Anotar las dificultades observadas

El objetivo de este instrumento de recolección de datos es el de recoger evidencia acerca de los aspectos involucrados en el proceso de enseñanza y aprendizaje en el contexto en que se ejerce, cuyo fin es llevar a cabo un análisis en las clases que reciben los estudiantes con discapacidad auditiva, para determinar las dificultades reales.

\section{Entrevista semiestructurada a docentes y estudiantes}

La entrevista es una técnica de gran utilidad en la investigación cualitativa para recabar datos; se define como una conversación 
que se propone un fin determinado distinto al simple hecho de conversar. (Diccionario de Ciencias de la Educación, Vol. 1. México: Santillana; 1983. p. 208.).

La entrevista a estudiantes busco identificar en los estudiantes con discapacidad auditiva, su percepción en cuanto a la educación que reciben en lengua escrita, y cómo influye en ellos en su rendimiento escolar y al socializar con sus compañeros de clase, amigos y familiares. $Y$ la entrevista dirigida a docentes es aplicada con el fin de Identificar las percepciones y conocimiento de los docentes en relación con la lectoescritura de los estudiantes con discapacidad auditiva.

Taller diagnóstico. En este instrumento se recoger mediante un taller práctico, evidencias acerca de las habilidades que tienen los estudiantes

Las orientaciones para aplicar este instrumento fueron:

-Se le dará una lámina al estudiante en la que se presentan 4 situaciones algunos personajes y objetos que están interactuando en un parque. Deberá contar todo lo que allí sucede en su primera lengua.

Se le presentarán 8 palabras. Deberá leer las palabras y decir cuál es la seña que le corresponde. Las palabras son palabras sencillas usadas en contextos familiares.

Se le da una a una las señas de las mismas palabras que se le presentaron anteriormente en la tarea de lectura y se le pide que la escriba en español una a una.

El educador especial marcará en la columna que aparece en un apartado del documento (Totalmente o Parcialmente), en el caso que el estudiante presente durante el taller; de lo contrario marcará (no lo hace) en el caso que el estudiante no presente durante el taller.

\section{Análisis y resultados}

Se emplea la triangulación como técnica con el fin de cruzar diversas estrategias aplicadas para la recolección de datos, Urquiza, A., Billi, M., \& Leal, T. (2017), en el su trabajo titulado Aplicar una distinción. Un programa sistémicoconstructivista para la investigación social cualitativa, se refiere a este tipo de triangulación de este modo: tiene que ver con el uso múltiple de métodos para estudiar un problema específico.. "Por ejemplo cuando se emplea la técnica de la entrevista como proceso inicial de recolección de información para luego ser contrastado con la observación participante y/o la discusión grupal" Pág.117.

Todo proceso investigativo lleva consigo un análisis minucioso en su etapa de recolección y análisis de datos recogidos mediante las técnicas $\mathrm{e}$ instrumentos. Para poder evaluar dichos instrumentos es contrastada las entrevistas semiestructuradas con la observación. Por esto es importante describir cualitativamente los resultados obtenidos, acorde al tipo de investigación que se realiza en el presente trabajo.

\section{Proceso de lectoescritura en estudiantes con discapacidad auditiva de media secundaria}

Durante la observación directa aplicada a estudiantes con discapacidad auditiva de los grados 10 y 11 , se pudo examinar que en el componente de Motivación e interés en la clase 12 de 16 estudiantes sordos no muestran interés a las actividades escritas propuestas por el docente, otros cuatro estudiantes demuestran este interés parcialmente. 
Ver Tabla.1

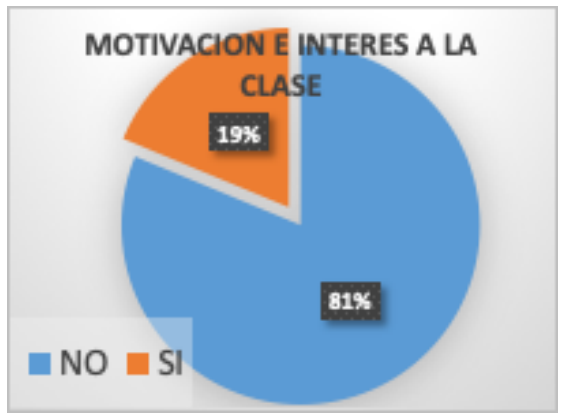

Fuente: elaboración propia (Imagen 2).

El total menos uno de los participantes no comprende por sí mismo las indicaciones que el docente les da por escrito lo cual trae consigo el solo 13 de los estudiantes respondan de manera entusiasta a la actividad escrita durante la clase y otros 3 estudiantes lo hagan parcialmente. Además, al analizar si contestaron acertadamente a las actividades escritas propuestas en clases arrojó como resultado que solo un estudiante lo hace parcialmente y los demás no lo lograban.

Tabla $\mathrm{N}^{\circ} 2$

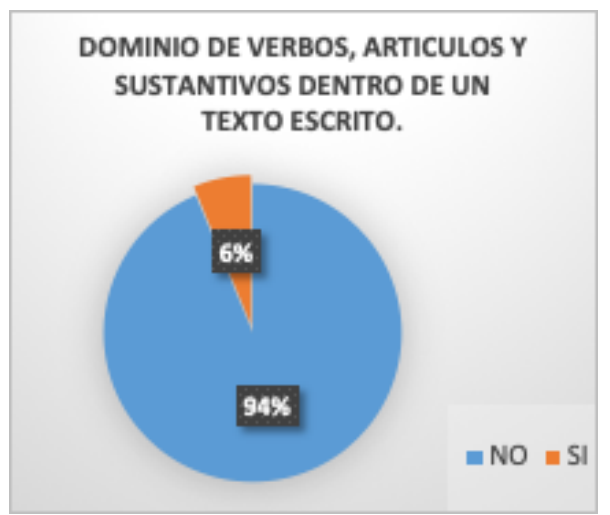

Fuente: Elaboración propia (Imagen 3).

Otro componente en la observación directa fue la ejecución de actividades y compromisos, los 16 estudiantes no lograron asimilar positivamente los compromisos propuestos por el docente, los cuales les exige al estudiante la investigación en fuentes de textos escritos.
Al presentar en clase los compromisos por escrito, solamente 11 de esta muestra lo hacía totalmente, aunque no entendían lo que plasmaron en sus tareas realizadas; dos de ellos transcribieron la tarea de sus compañeros y los otros tres no presentaron el compromiso. Esto trajo como consecuencia el no poder participar en clase de forma activa de acuerdo al compromiso previamente escrito.

Tabla $N^{\circ} 3$

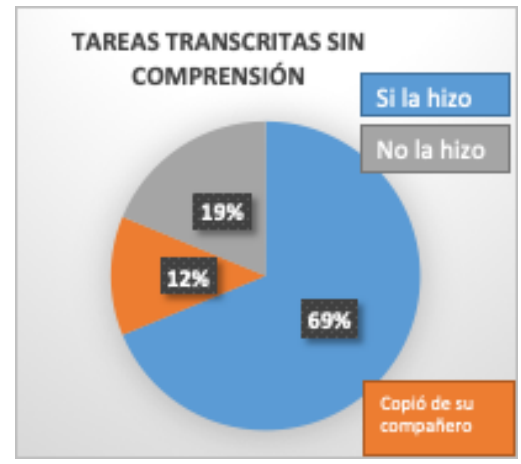

Fuente: Elaboración propia. (Imagen 4).

Por último, en este instrumento se analizó el desarrollo de la clase por el docente, la cual, si demostró totalmente empatía hacia los estudiantes sordos, al emplear estrategias pertinentes para los sordos siente dificultad, solo se apoyó en el intérprete, aun así genera totalmente confianza hacia los estudiantes y el uso de herramientas tecnológicas en su clase y dinámicas no fue notable.

Otro instrumento y técnica de recolección de información fue una entrevista semiestructurada a estudiantes sordos de grados 10 y 11 , que tenía como objetivo identificar en los estudiantes con discapacidad auditiva su percepción en cuanto a la educación que reciben en lengua escrita y cómo influye en su rendimiento escolar y al socializar con sus compañeros de clase, amigos y familiares, el resultado fue el siguiente: 
Los 16 estudiantes coincidieron en que las habilidades que tienen en la lectoescritura no son las suficientes para comunicarse de manera escrita con su entorno y eso influye grandemente en la interacción con sus docentes, compañeros y amigos oyentes. expresan que durante la clase de lengua castellana se sientes desinteresados y perdidos, no logran comprender fuentes con textos escritos para responder a tareas propuestas por el docente y tampoco encuentran esta información en su mismo idioma.

Han tenido en sus manos libros, revistas, periódicos entre otros textos, pero solo pueden identificar algunas palabras de ellos o guiarse de la información expuesta en imágenes que contienen. Es cuando ven la importancia de adquirir la habilidad de lectura y escritura para no solo acceder a ellos sino también el poder producir escritos, ya sean comedias, terror o ficción que son los géneros más deseados por este grupo intervenido.

En la prueba diagnóstica que se le aplica a este grupo de estudiantes demuestran interés y capacidad para interiorizar y describir de manera correcta las imágenes que se les presentó usando su primera lengua. Aunque al momento de leer y entender las oraciones propuestas en español escrito se les dificulta, aún más al identificar palabras para asociarlas con su respectiva seña.

Por otro lado, en el componente de escritura de este instrumento, el grupo en general no logran entender la seña y escribirla totalmente, y no la emplean correctamente dentro de una oración.

\section{Estrategias didácticas en estudiantes con discapacidad auditiva de media secundaria}

Los estudiantes dejaron como sugerencia a los docentes tener en cuenta más estrategias o dinámicas en las clases, apoyándose mucho en imágenes ya que dicen ser muy visuales; además que interactúan mucho más con ellos y logren aprender más lengua de señas para no depender en la comunicación sólo del intérprete, siendo más directo el acercamiento entre estudiantes sordo y docente.

La entrevista semiestructurada aplicada a 4 docentes de áreas como español, Matemáticas, Informática y Ciencias Naturales comentaron que faltan métodos de enseñanza del español como segunda lengua y que los docentes no tienen claro los conceptos de lectura, escritura y alfabetización de estudiantes sordos. Todo esto acompañado de la carencia de recursos visuales, tecnológicos que se están desaprovechando en este siglo $\mathrm{XXI}$, como video beam, herramientas gráficas. No es suficiente con los libros de vocabularios académicos en LSC sino también con videos en lengua de señas que ayude a potencializar su idioma y además aprender un vocabulario del español. Aunque se tiene buena disposición tenemos el tiempo y queremos hacerlo de la forma correcta pienso que se hace de una forma equivocada porque no tenemos pasos puntuales $u$ orientaciones puntuales para que ellos mejoren sus prácticas educativas.

\section{Material didáctico multimedia}

Los docentes y estudiantes entrevistados mencionan claramente la necesidad que existe en el uso de las tecnologías mediante estrategias didácticas en el proceso de enseñanza, carente en la institución. lo cual se convierte en una debilidad al no verse soportado todo el caudal de conocimientos y saberes que el docente debe impartir en los estudiantes con discapacidad auditiva.

En esta investigación se ha observado que existe de forma general una problemática desde 
el que hacer pedagógico en la estimulación y enseñanza de la lengua escrita en estudiantes sordos. Lo cual afecta el buen desempeño académico e interacción con sus pares y todo su entorno, limitándose a solamente obtener información a través de la lengua de señas, de esta forma se aleja el sistema educativo hoy día de la propuesta Educación Bilingüe que se plantea en el decreto de Inclusión vigente 1421 del 2017.

\section{Propuesta pedagógica}

Se presenta una propuesta pedagógica, resultado de todo un proceso de observación, indagación, análisis y recolección de datos, desde la inmersión en la cotidianidad escolar de los sujetos o participantes, dando respuesta a la situación problema planteada anteriormente. Consiste en un material multimedia interactivo, apoyado en la metodología "Kamishibai", que emplearon inicialmente en Japón y luego en otros países para la enseñanza de una segunda lengua a niños y jóvenes de una forma dinámica y experiencial.

Al surgir la necesidad de una adaptación del Kamishibai a la Lengua de señas, mediante una interfaz interactiva, la cual presenta videos y actividades en el idioma de los estudiantes sordos para fortalecer la lectura y escritura, nace la propuesta innovadora de SÓDORI, palabra en japonés que traduce "SORDO LEE".

Sódori es el inicio de un software atractivo a estudiantes sordos, que reúne todo el proceso de enseñanza de la lengua escrita sugerida por el Instituto Nacional de Sordos de Colombia, acercándose a la realidad de la situación para así fortalecer la lectura y escritura mediante narrativas, imágenes y actividades que ayudan a poner en práctica las lecciones, permitiendo una autoevaluación y seguimiento de los mismos estudiantes.
SÓDORI es una vena arteria de Kamishibai, que se destaca por ser un material multimedia didáctico adaptado a lengua de señas, convirtiéndose en el primer Kamishibai registrado con estas características y como recursopedagógicoy didácticodaoportunidades para ampliar la mirada multicultural, despertar el deseo de crear relatos, disfrutarlos en grupo, abordar diversidad de obras, hacer integración curricular entre distintos saberes propios de subsectores como la educación artística, la música, la educación tecnológica, etc., de acuerdo a lo también planteado por Carreño, L. C.(2012). Desarrollo del lenguaje y uso del Kamishibai. Cuadernos de Educación de la Universidad Alberto Hurtado, 44, 1-7.

El Kamishibai ha sido usado en contextos escolares como por ejemplo el profesor Velarde Herrera (2014) y concluyó que los procesos en la adquisición de una lengua extranjera, se ven favorecidos en los ambientes cuidados que transmiten sensaciones de seguridad y afecto para/con los niños y las niñas; es por ello que el profesorado necesitará apoyarse en diversos recursos para la animación lectora.

Sódori, es un material que se ajusta a las necesidades de estudiantes de esta Institución, atendiendo a los intereses y resultandos atractiva para cada joven que lo use, aumentando cada vez más el deseo por adquirir la segunda lengua (español escrito).

Las tecnologías de la información y de la comunicación son particularmente importantes para las personas con discapacidad auditiva: son relativamente fáciles de acceder, usan, por lo general, el canal de percepción visual y los textos, así que permiten a las personas sordas participar en diferentes situaciones sociales y educativas (Agboola y Lee, 2000). Por esta otra razón, Sódori se convierte en una herramienta apoyada en las Tics para la fortalecer la lectura y escritura en estudiantes 
sordos. El cual tiene como Objetivo general: Fortalecer el proceso de lectoescritura en estudiantes con discapacidad auditiva. Dentro de sus Objetivos específicos tiene: Emplear el Kamishibai durante las clases de Lengua escrita de los estudiantes con discapacidad auditiva. Relacionar textos escritos interpretados a lengua de señas. Enseñar un vocabulario en español escrito. Completar oraciones escritas y apareamiento de palabra-seña, con el vocabulario previamente enseñado.

Es así como el Kamishibai adaptado a la población con discapacidad auditiva, pretende fortalecer el proceso de Lectoescritura en los estudiantes sordos, proporcionando esta herramienta didáctica a los docentes para ejecutarlo de manera creativa a los jóvenes, favoreciendo la interacción de estos con el entorno. La aplicación de los instrumentos arrojó un resultado valioso para la elaboración del contenido del multimedia que se está construyendo, y fue el interés a la lectura de textos narrativos, dramáticos, cómicos y de terror. Demostró también el deseo de estos estudiantes a superarse adquiriendo habilidades en la segunda lengua para desenvolverse de mejor manera en la sociedad, teniendo un nuevo canal de acceso a la información y comunicación.

Una vez elaborado y aplicado el material multimedia aquí propuesto, se espera una mejora progresiva en las competencias de lectura y escritura, desarrollando un máximo interés en las áreas lo cual repercute en la calidad del aprendizaje y notas. Además de esto, el desarrollo en la dimensión cognitiva de estos estudiantes se verá enriquecida.

Sódori presenta un hilo secuencial de acciones al igual que el ciclo del diseño de una clase en el aula con estudiantes sordos, en donde se tiene un objetivo principal presentando un tema junto a una evaluación diagnóstica individual de saberes previos de cada participante, para luego mostrar los materiales y método para ejecutar las posteriores actividades en donde se implementa lo aprendido y a la vez se hace la evaluación del mismo tema planteado desde un inicio.

1. Presentación del tema: el estudiante sordo selecciona la narrativa que desea estudiar y observa cuantas veces sea necesario el video en lengua de señas.

2. Evaluación previa: al continuar, navegando con la flecha de la interfaz que indica Siguiente, puede observar el mismo texto, pero en español escrito y al lado de la pantalla encontrará un cuadro en blanco para digitar ahí las palabras que le sean conocidas. Dichas palabras se cargarán automáticamente en un bloc de notas del computador. Esto con la finalidad de que el docente encargado pueda evaluar el saber previo del estudiante en cuanto a la segunda lengua, específicamente.

3. Materiales y método: en esta parte, encontrará un listado de palabras preseleccionada del mismo texto escrito acompañadas de su equivalente en lengua de señas colombiana, para efectos de que el estudiante pueda relacionarlas inmediatamente y aprenderlas.

4. Actividades y evaluación: cada narrativa tiene 3 actividades interactivas de unir palabras, completar oraciones y armar oraciones. Cada actividad tiene la posibilidad de ser medida o calificada por estrellas según las respuestas acertadas o equivocadas. Solo avanza a la siguiente actividad si completa las respuestas de manera correcta. De esta manera se realiza una evaluación constante del aprendizaje.

Teniendo en cuenta la capacidad máxima de los estudiantes para las clases, es recomendable no saturarlos con muchas actividades, así es más productiva la clase. 
Imagen $\mathrm{N}^{\circ} 5$. Propuesta Didáctica
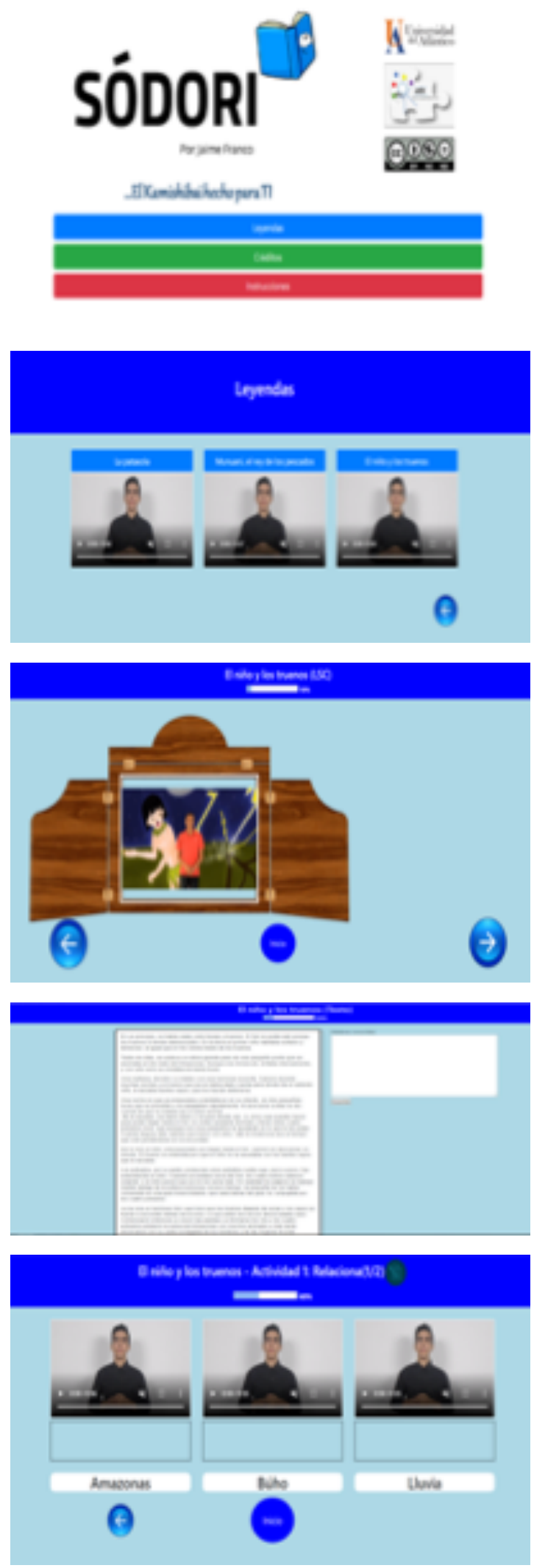
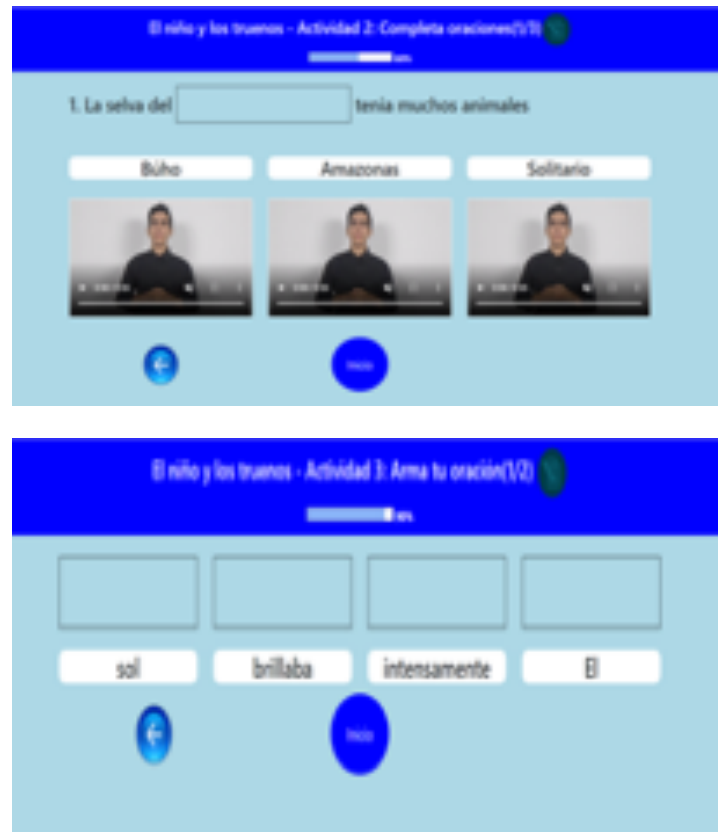

Fuente: elaboración propia de autores (Imagen $5)$.

\section{Evaluación y seguimiento}

El éxito de este material se alcanza gracias a las pruebas, evaluaciones y observaciones de un personal experto en tecnología, inclusión y pedagogía aplicada a personas sordas, a continuación, se presenta sus apreciaciones u observaciones en cuanto a Sódori:

En la prueba piloto, se recoge la siguiente observación de los estudiantes sordos:

Sódori, permitió estimular el interés al proceso de lectoescritura en los estudiantes sordos, proporcionando esta herramienta didáctica a los docentes para ejecutarlo de manera creativa a los jóvenes, favoreciendo la interacción de estos con el entorno. En la aplicación de Sódori se destaca el interés a la lectura de textos narrativos, dramáticos, cómicos y de terror. Demostró también el deseo de estos estudiantes a superarse, adquiriendo habilidades en la segunda lengua para desenvolverse de mejor manera en la sociedad, teniendo un nuevo canal de acceso a la información y comunicación. 


\section{Conclusiones}

Los resultados de esta investigación van en concordancia con lo que expone Patricia M. Folco (2010), al señalar que el ámbito escolar debe propulsar la utilización de los recursos de información y comunicación para la construcción de significado. Y es precisamente lo que presenta Sódori, una herramienta escolar adaptada a estudiantes sordos para ofrecer información, construyendo conocimientos que les serán útil en su vida.

Por otro lado, Lilia Concha Carreño de la Universidad Alberto Hurtado en Chile, menciona que el Kamishibai ofrece la oportunidad de abrir diálogo y conversación posterior a la narración, propicia las relaciones sociales, favorece la comunicación oral, ayuda a crear un ambiente de confianza, acogedor y lúdico que motiva la expresión personal.

Durante la aplicación de este material, se reflejó el aumento de la participación en clase de estudiantes sordos, al ser motivado el aspecto de la comunicación y expresión oral, así como también la habilidad para socializar, refutar y defender posiciones o puntos de vista de cada quien.

Otro aspecto importante y muy valioso para el éxito de esta investigación lo es el uso de las tecnologías, Cabero (2000), hace alusión a esta herramienta que permite el acceso, desarrollo, tratamiento y procesamiento de la información, sin el límite condicionante que imponen las barreras espacio temporales y con el beneficio adicional de potenciar en buena medida, la interactividad y el asincronismo entre los actores educativos de cada plantel formativo.

1. En la revisión teórica, se fundamenta de las categorías de este tema de investigación y observación del estudio problema, se puede afirmar que existe una lista de información e investigaciones que aportan a este tema, dejando claro que la población de estudiantes con discapacidad auditiva se encuentra en desventajaen comparacióncon suscompañeros oyentes al momento de comunicarse de forma escrita con su entorno. Por esta razón, en los estudios encontrados hay bajo rendimiento académico por este tipo de estudiantes sordos, poca motivación al estudio entre otros factores.

2. Otra realidad encontrada, es el reto al que se enfrenta el docente, por lo que se hace necesario idear herramientas, estrategias, dinámicas que faciliten el proceso de enseñanza y motivación al estudio de estos estudiantes sordos.

3. Sódori, generó un impacto visual, emocional y de conocimiento a los estudiantes sordos, de tal manera que cautivó su interés gracias a la interfaz, actividades y videos ahí expuestos. Lograron entender y usar de manera fácil el material multimedia, realizando las actividades ahí planteadas. Lo más importante fue la pertinencia del contenido, al tener temas de narrativas de su interés.

4. La experiencia deja claramente expuesto que el uso de las tecnologías juega un papel importante en la educación de los estudiantes hoy día; al ser empleada en el aula de clase, el docente entra en "órbita" con sus estudiantes sordos, los de esta generación, estableciendo un vínculo mucho más dinámico, motivador propicio para la enseñanza y aprendizaje, logrando de manera más fácil alcanzar logros en cada uno de los estudiantes a su ritmo. 


\section{Referencias bibliográficas}

Agudelo, M., \& de Jesús, W. (2012). Las TIC como herramientas potenciadoras de equidad, pertinencia e inclusión educativa (Information Technology and Communication Empowering Tools Equity, Relevance and Educational Inclusion). Trilogía Ciencia Tecnología Sociedad, 4(7).

Álvarez, H. O., \& Ramírez, D. A. (2006). Experiencias e investigaciones sobre las TIC aplicadas a la atención de personas con necesidades educativas. Citado de Libro 3. Las tecnologías de la información y la comunicación (TIC): Avances, retos y desafíos en la transformación educativa. (2015). Recuperado de https://www.transformacion-educativa.com/attachments/ article/137/Libro\%2003\%20-\%20Las\%20tecnolog\%C3\%ADas\%20de\%20la\%20informaci\%C3\%B3n\%20y\%20la\%20comunicaci\%C3\%B3n.pdf

A. M. Colmenares, (2012). Investigación-acción participativa: una metodología integradora del conocimiento y la acción. Voces y Silencios: Revista Latinoamericana de Educación, Vol. 3, No. 1, 102-115 ISSN: 2215-8421. Citado de Díez-Gutiérrez, E.-J. (2020). Otra investigación educativa posible: Investigación-acción participativa dialógica e inclusiva. Márgenes Revista De Educación De La Universidad De Málaga, 1(1), 115-128. https://doi.org/10.24310/ mgnmar.v1i1.7154

Bernal Velásquez, A. L. (2018). Estrategias de enseñanza para maestros con niños sordos, del Instituto Nuestra Señora de la Sabiduría. Recuperado de https://bdigital.uexternado.edu. co/handle/001/852

Buitrago López, C. C. (2017). Representaciones sociales de un grupo de personas sordas adultas frente a la cultura escrita. Recuperado de http://www.cultura-sorda.org/reflexiones-sobre-la-escritura-y-la-alfabetizacion-de-los-ninos-sordos/

http://bibliotecadigital.univalle.edu.co:8080/bitstream/10893/5546/1/REFLEXIONES\%20 ACERCA.pdf

Chóez Jaramillo, H. G., \& Arreaga Suárez, M. G. (2019). Lenguaje de señas en el desarrollo emocional de las personas sordas (Bachelor's thesis, Universidad de Guayaquil. Facultad de Filosofía, Letras y Ciencias de la Educación.). Recuperado de http://repositorio.ug.edu. ec/handle/redug/44920

Concha Carreño. L (2012). Desarrollo del lenguaje y uso del Kamishibai. Académica Universidad Alberto Hurtado Chile. Cuaderno de Educación N 44, junio de 2012.

Recuperado de http://mailing.uahurtado.cl/cuaderno_educacion_44/pdf/articulo44_kamishi. pdf 
Cruz-Aldrete, M. (2008). El estudio de las lenguas de señas. Los sordos, ¿hijos de un dios menor? Signos lingüísticos. Citado por Nieto Martin. (2017). El Aprendizaje de la Gramática Española por parte de Aprendientes Extranjeros y Sordos: Puntos de Encuentro. Revista Hechos y Proyecciones del Lenguaje. Vol. 23 (2017). Recuperado de https://revistas.udenar. edu.co/index.php/rheprol/article/view/3841

García, A. M. M. (2015). El español escrito como segunda lengua en el sordo: apuntes para su enseñanza. Boletín de Lingüística, 27(43-44), 118-131.

García Amaya. (2017). Las TIC como herramientas potenciadoras de equidad, pertinencia e inclusión educativa. Trilogía Ciencia Tecnología Sociedad, (7), 61-77. Recuperado de https:// repositorio.uptc.edu.co/bitstream/001/2326/1/TGT-967.pdf

Gómez, H. M. R. (2017). Desde el universo de las letras. Agenda Cultural Alma Máter, (245). Recuperado de file:///C:/Users/Jaime\%20Franco/Downloads/328709-Texto\%20del\%20art_ culo-130903-1-10-20170817.pdf

González, Y., Lora, F., Rendón, L., Saldarriaga, C., Consuegra, J., \& Franco, J. (2007). Aproximaciones a la enseñanza de la lengua escrita como segunda lengua en las personas sordas. Bialfabetismo: Lectura y escritura en dos lenguas en Colombia, 119-133. Citado por Tobar, L. (2017). La definición en la lengua de señas colombiana (LSC). Universidad del Valle Santiago de Cali, Colombia. Recuperado de http://www.scielo.org.co/pdf/leng/v45n2/01203479-leng-45-02-00383.pdf

Gutiérrez, M. E., Ball Vargas, M. S., \& Márquez, E. (2008). Signo, significado e intersubjetividad: una mirada cultural. Educere, 12(43), 689-695.). citado de Espinosa, R. Gonzales, L. \& Hernandez, R. (2019). Implicaciones de la reflexión y la mediación didáctica en docentes en formación durante su práctica educativa. Revista Scielo, versión no.34.

Recuperado de http://dx.doi.org/10.14483/23448350.13442

Henao Álvarez, O., Ramírez Salazar, D. A., \& Medina Medina, M. (2004). Potenciando la capacidad lectora de los sordos con el apoyo de nuevas tecnologías. Virginia, Hael. (2018). Abordajes de la Lectura por parte de Sordos en Contextos Bilingües en Países de Habla Hispana. Revista latinoamericana de educación inclusiva. vol.12 no.2 Santiago nov. 2018. Recuperado de http://dx.doi.org/10.4067/S0718-73782018000200079

Hernández, I. (2012). Investigación cualitativa: una metodología en marcha sobre el hecho social. Citado de Rodríguez, F. Caurcel, Cara. (2019). Aproximación cualitativa del escudriño en Psicología educativa. Recuperado de http://dx.doi.org/10.20511/pyr2019.v7n1.301

Herrera, V. (2005). Habilidad lingüística y fracaso lector en los estudiantes sordos. Citado de 
Rodriguez, H. (2017). Influencia De La Competencia Metafórica En Lengua De Señas Sobre La Comprensión Lectora En Un Grupo De Estudiantes Sordos Con Estilo Cognitivo Documentado. Universidad Pedagógica Nacional. Recuperado de http://repository.pedagogica. edu.co/bitstream/handle/20.500.12209/7652/TO-21332.pdf?sequence=1\&isAllowed=y

Zuniga Palma, M. J. (2018). Implementación de las Tic' s en la enseñanza de la matemática como recurso pedagógico en los estudiantes de educación básica superior de la Unidad Educativa Fiscal" Dr. Emilio Uzcátegui García" de la parroquia Pascuales cantón Guayaquil, durante el período lectivo 2017-2018 (Doctoral dissertation).. Recuperado de http://biblioteca.uteg.edu.ec/xmlui/handle/123456789/182

Franco. J. (2019). Material didáctico multimedia para el fortalecimiento de la lectoescritura en estudiantes con discapacidad auditiva. Universidad del Atlántico.

Franco, J; Barreiro, S. (2019). Kamishibai adaptado. (Imagen 1). Universidad del Atlántico. Franco, J; Barreiro, S. (2019). Motivación e interés a la clase. (Imagen 2). Universidad del Atlántico.

Franco, J; Barreiro, S. (2019). Dominio De Verbos, Artículos Y Sustantivos Dentro De Un Texto Escrito. (Imagen 3). Universidad del Atlántico.

Franco, J; Barreiro, S. (2019). Tareas Transcritas Sin Comprensión. (Imagen 4). Universidad del Atlántico.

Franco, J; Barreiro, S. (2019). Propuesta Didáctica. (Imagen 5). Universidad del Atlántico.

Lissi, M. R., Svartholm, K., \& González, M. (2012). El Enfoque Bilingüe en la Educación de Sordos: sus implicancias para la enseñanza y aprendizaje de la lengua escrita. Estudios pedagógicos (Valdivia), 38(2), 299-320.

Liaño Gutiérrez, A. (2018). La lectoescritura en personas con discapacidad auditiva.

MEN (2008). Educación inclusiva con calidad "construyendo capacidad institucional para la atención a la diversidad" Guía y Herramienta No 34. Recuperado de https://www.researchgate.net/publication/314151626_educacion_inclusiva_con_calidad_construyendo_capacidad_institucional_para_la_atencion_a_la_diversidad_Guia_y_Herramienta

Morales, A. M. y Fraca de Barrera, L. (2000). Hacia una política educativa para la enseñanza y el aprendizaje de la lengua escrita en sordos. Trabajo de grado de maestría no publicado, Universidad Pedagógica Experimental Libertador, Instituto Pedagógico de Caracas.

Lugo Martínez, J. (2019). Resignificando la educación inclusiva y la multiculturalidad en el Colegio Helvetia. Recuperado de http://hdl.handle.net/10554/46028

Proyecto Kamishibai en el I.E.S. Ribera del Arga - Curso 2009/2010. Navarra, España. Recuperado de https://iesriberaargakamishibai.wordpress.com/2010/05/17/34/ 
Rangel, M. T. (2007). El proceso de la escritura en estudiantes adolescentes sordos. Recuperado de http://www.modalidadespecial.educ.ar/datos/recursos/pdf/el-proceso-de-escritura-en-adolescentes-sordos.pdf

Rincón-Bustos, M. L., Aguirre-Bravo, Á., Carmona, S. M., Contreras-Ruiz, P., Figueredo-Higuera, L., Guevara-Urrego, C., ... \& Urán-Loaiza, A. J. (2015). ¿Cómo la comprensión de lectura en estudiantes sordos se ve facilitada por el uso de tecnologías de la comunicación e información? Revista de la Facultad de Medicina, 63(3Sup), 83-91.)

Reyes Cortes, F. (2013). Las TIC un medio seguro hacia el dominio del español como segunda lengua en sordos (Bachelor's thesis, Facultad de Educación).

RODRÍGUEZ HERNÁNDEZ, H. J. (2020). Profesores Inclusivos: una Propuesta de Formación Inicial. Recuperado de http://hdl.handle.net/10953/

Rojas Gil, A. M. (2005). Representaciones sociales de un grupo de estudiantes sordos frente a la lecto escritura (Master's thesis). Recuperado de https://repository.cinde.org.co/bitstream/handle/20.500.11907/1889/RojasGil_2005.pdf?sequence=1

Skliar, Carlos - La educación de los sordos- 2003 recuperado de http://www.espaciologopedico.com/articulos2.php?ld_articulo=305

Solano, C. I. H., \& Barraza, J. A. V. (2018). Sordera y Lectura: Un análisis histórico e interconductual (Vol. 1). StaUdeG/Universidad de Guadalajara. Recuperado de https://books. google.es/books?hl=es\&lr=\&id=fPRgDwAAQBAJ\&oi=fnd\&pg=PA79\&dq=Schimmel,+Edwards $+y+$ Prickett+(1999)+reportan+que+estudiantes+de+18+a\%C3\%B1os+tienen+un+desempe\% C3\%B10+lector+promedio+equivalente+al+observado+en+el+cuarto+grado+en+la+escuela+norteamericana\&ots=7dbJKri46Q\&sig=Geq8F87AsMoZR96n_LkMGMub3q8\#v=onepage \&q\&f=false

Sosa, L. R. P. (2018). Destrezas de lectoescritura en estudiantes preadolescentes sordos puertorriqueños (11 a 13 años) (Doctoral dissertation, Universidad del Turabo). Recuperado de https://gurabo.uagm.edu/sites/default/files/uploads/Health-Sciences/Thesis/2018/Elisamuel-Rivera-PHL-2018.pdf

UNESCO, Estándares de competencias en tic para docentes, recuperado de http://www. eduteka.org/pdfdir/UNESCOEstandaresDocentes.pdf Londres, enero 8 de 2008

Saquete, E., Vázquez, S., Lloret, E., Llopis, F., Gómez, J. M., \& Mosquera, A. (2013). Tratamiento de textos para mejorar la comprensión lectora en alumnos con deficiencias auditivas. Procesamiento del lenguaje natural, (51), 231-234. 
Silva Lascano, C. A. (2017). Aplicaciones informáticas como apoyo a la comunicación de los estudiantes con discapacidad auditiva de la Unidad Educativa Especializada "Dr. Camilo Gallegos" del cantón Ambato, provincia de Tungurahua (Bachelor's thesis, Universidad Técnica de Ambato. Facultad de Ciencias Humanas y de la Educación. Carrera de Docencia en Informática). Recuperado de https://repositorio.uta.edu.ec/jspui/handle/123456789/25690

Tovar, L. (2002). Un enfoque interdisciplinario para la enseñanza de la lengua escrita a niños sordos. Citado por Tobar, L. (2017). La definición en la lengua de señas colombiana (LSC). Universidad del Valle Santiago de Cali, Colombia. Recuperado de http://www.scielo.org.co/ pdf/leng/v45n2/0120-3479-leng-45-02-00383.pdf

Tovar, Lionel. (2000). La lengua escrita como segunda lengua. En: Bilingüismo de los sordos. INSOR. Vol 11 N.4. Santa Fé de Bogotá. Pág. 74-87. Citado por Tobar, L. (2017). La definición en la lengua de señas colombiana (LSC). Universidad del Valle Santiago de Cali, Colombia. Recuperado de http://www.scielo.org.co/pdf/leng/v45n2/0120-3479-leng-45-02-00383.pdf

Triana Varela, Y. P. (2019). El proceso de escritura a través del juego simbólico.

UNESCO (2008). Conclusiones y recomendaciones emanadas de la 48a reunión de la Conferencia Internacional de Educación. Ginebra, Suiza: UNESCO-OIE. Recuperado de http:// www.ibe.unesco.org/es/cie/48a-reunion-2008.html

Utria-Machado, L. (2017). Actitudes de jóvenes sordos de básica secundaria hacia el proceso de escritura. Revista Colombiana De Rehabilitación, 16(1), 24-31.

Valdez, M. D. R. G. (2020). Beneficios del uso de las nuevas tecnologÃas de la informaci $\tilde{A}^{3} n$ como herramienta en la docencia/The Benefits of the Usage of New Information Technologies as a Tool for Teaching. Revista lberoamericana de Producci $\tilde{A}^{3} n$ AcadÃ@ mica y Gesti $\tilde{A}^{3} n$ Educativa, 6(12). Recuperado de https://www.pag.org.mx/index.php/PAG/article/view/823

Vasilachis de Gialdino, I. (2019). Estrategias de investigación cualitativa. Madrid: Editorial Gedisa. Recuperado de http://www.ceil-conicet.gov.ar/2019/05/libro-estrategias-de-investigacion-cualitativa-volumen-ii-irene-vasilachis-de-gialdino-coord/

Velarde Herrera J. (2014). La animación lectora en contextos bilingües: el Kamishibai como recurso. Universidad de Valladolid, Facultad de Educación. Palencia, España 2014.

Yovera Sandoval, E. C. (2018). Los materiales didácticos y su influencia en la formación de la identidad cultural regional de los alumnos de tercer año de Educación Secundaria de la Institución Educativa Parroquial "Santa Ana", Huarmaca-2018. Recuperado de http://repositorio.uladech.edu.pe/han 